\title{
ENHANCED SECURE INTERFACE FOR A PORTABLE E-VOTING TERMINAL
}

\author{
André Zúquete \\ IEETA, University of Aveiro, Campus Univ. de Santiago, 3810-193 Aveiro, Portugal \\ andre.zuquete@ua.pt
}

Keywords: $\quad$ E-voting, user interface, privacy, visual authentication, usability

\begin{abstract}
This paper presents an enhanced interface for an e-voting client application that partially runs inside a small, portable terminal with reduced interaction capabilities. The interface was enhanced by cooperating with the hosting computer where the terminal is connected to: the hosting computer shows a detailed image of the filled ballot. The displayed image does not convey any personal information, namely the voter's choices, to the hosting computer; voter's choices are solely presented at the terminal. Furthermore, the image contains visual authentication elements that can be validated by the voter using information presented at the terminal. This way, hosting computers are not able to gather voters' choices or to deceive voters, by presenting tampered ballots, without being noticed.
\end{abstract}

\section{INTRODUCTION}

Internet voting systems are appealing for several reasons, one of them being the mobility of voters. Using the Internet, voters may contact the right electoral servers virtually from anywhere in the world. However, the computers used by voters to express their will must be trusted. Namely, they should not steal authentication credentials, link votes to voters or interfere destructively with the voting process. In other words, voters should interact only with a trusted application, running on top a secure platform - trusted computing base, TCB - when expressing their vote. However, this goal is hard to achieve. Nevertheless, this "secure platform problem" is a fundamental problem that needs to be solved when trying to design remote electronic voting systems.

In the context of the Robust Electronic Voting System (REVS (Joaquim et al., 2003)) was developed an intrusion-tolerant voting client using a TCB composed by a FINREAD portable terminal and a smartcard (Zúquete et al., 2007). The terminal provides protection against disclosure for user input (authentication secrets, voter's choices) and output (presentation of voter's choices). Furthermore, it provides output authentication for the presented ballot. With this TCB, a voter may securely use any hosting computer to access REVS electoral servers in order to vote in a particular election. However, the line-based interface of a FINREAD terminal is too reduced to give a clear, global view of the ballot and the voter choices. This issue is particularly relevant when ballots have many, long questions, each with several possible answers.

This paper describes an enhancement of the interface between the TCB and the voter to facilitate voting processes. To enhance the interface, we extended the functionality of the voting terminal for cooperating with a hosting computer in showing images of ballots being filled. However, since we cannot trust hosting computers for keeping secret the choices expressed by voters, the images presented by hosting computers cannot contain any reference to voters' choices. One way to achieve this goal could be to use visual cryptography (M. and Shamir, 1995), but this technique is not convenient for voters, raises many operational problems and creates coercion vulnerabilities. Alternatively, we used directly readable text for helping voters to get a clear view of their choices, but in such a way that no relevant, personal information if leaked for hosting computers.

Furthermore, the presented image should be immune to modifications introduced by hosting computers, which requires their visual authentication by voters. This means that either (i) the voter sees the correct ballot or (ii) the voter sees a tampered image but the tampering action is clearly perceptible by the voter. The solution adopted allows the voter to repeatedly check the integrity of the displayed image using contents simultaneously presented on the terminal display. 


\section{REVS VOTING SYSTEM}

REVS is a blind-signature based voting system designed for providing secure and robust electronic voting using the Internet (Joaquim et al., 2003). The system has a client application (Voter Module) that conducts the interaction with a set of electoral servers. To improve the privacy of the voter and accuracy of his participation in the election, the Voter Module should use a TCB for protecting some critical data and voter-computer interactions. Concerning the user interface, the TCB must provide:

- Trusted output: correctly present to the voter authenticated ballots provided by electoral servers.

- Protected input: securely get all input from the voter - authentication secrets and ballot choices.

The TCB was implemented with a smartcard and a FINREAD (FINREAD Consortium, 2003) reader, which has I/O capabilities (Zúquete et al., 2007). Fig. 1 shows the new architecture of REVS using this TCB. FINREAD terminals are embedded systems capable of hosting different Java applets (Finlets) and a JVM to execute them. A FINREAD device has reduced human-machine I/O capabilities: it has a small LCD display, with 4 lines of 20 characters, and a 16key pin-pad (see Fig. 2). Finlets control all interactions with the smartcard and can use the terminal I/O capabilities to perform protected input/output with the terminal user (e.g. get the smartcard PIN).

One of the tasks of the Voter Module Finlet is to deal with the interface with voter, namely with the presentation and filling of ballots. Ballots are signed XML documents fetched from electoral servers and validated by voters using their smartcard. If valid, a ballot is presented and filled using the FINREAD display and pin-pad. The part of the Voter Module that runs in the hosting computer has no direct influence in the presentation and filling of the ballot.

\subsection{FINREAD Human Interface Issues}

Due to the FINREAD display limitations, the presentation of the ballot in this device was completely changed from the original REVS model. Furthermore, the ballots' text to present to voters must be produced differently by the electoral Authorities, namely using reduced amounts of text in questions an answers taking into consideration the reduced capabilities of a FINREAD display. Nevertheless, the FINREAD display is too reduced for having a clear and complete view of the ballot and voter's choices. This limitation can be a major constraint when filing long ballots, with many questions and answers.

To overcome the physical output limitations of the FINREAD terminal we considered a solution based on cooperating with the hosting computer: the terminal produces an image of the filled ballot and sends it to the Voter Module for being displayed at the hosting (a) REVS original architecture

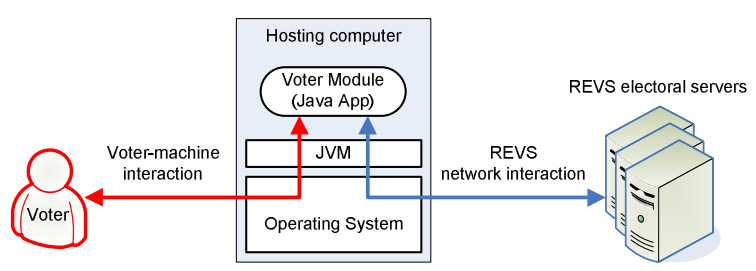

(b) REVS architecture with a smart card/FINREAD TCB

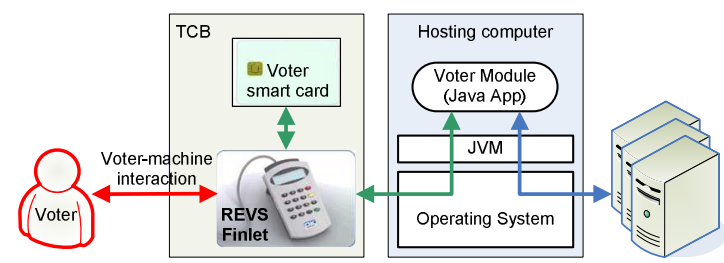

Figure 1: Evolution in the architecture of REVS for supporting a smartcard/FINREAD TCB at the voter side.

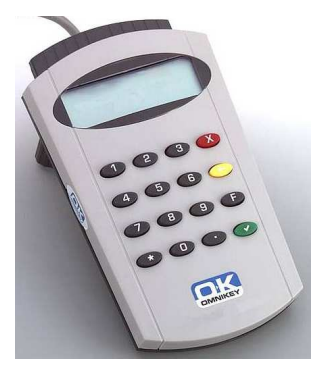

Figure 2: FINREAD terminal from Omnikey.

computer. But for producing these images we have two critical requirements:

- The displayed image should not disclose any useful information to others than the voter.

- The displayed image should allow the voter to easily detect tampering actions performed by the Voter Module and/or the hosting computer.

The first requirement is critical for the confidentiality of voters choices. Otherwise, a malicious hosting computer (or Voter Module) could store the images of the ballots presented with voters' choices. Furthermore, images should not enable attackers to coerce voters to prove how they have voted.

The second requirement is critical for preventing a malicious hosting computer from presenting a tampered ballot, which could mislead voters in many ways by (i) modifying questions, (ii) changing possible answers or (iii) changing chosen answers.

In the next section we discuss related work regarding the secure presentation of contents (images) produced by our TCB. Afterwards, we describe some usability issues of voting systems are their impact in our system. Then, we describe our contribution to enhance the interface of the voting terminal. 


\section{RELATED WORK}

Visual cryptography (M. and Shamir, 1995) allows humans to decrypt an image using another image, printed in a transparency. The encrypted image is presented in the screen, or printed in a sheet of paper, and the decryption transparency, a slide with a painted decryption pattern, must be placed on top of it to turn an area filled with apparently random dots into something understandable by a human. Visual cryptography can also be used for content integrity; (Naor and Pinkas, 1997) discusses several visual authentication methods, using visual cryptography, for any kind of visual data: numerical, textual or graphical.

Visual cryptography could be used for ensuring the privacy and integrity control of the ballot images presented by hosting computers; the key to encrypt the image could be entered with the FINREAD pin-pad. However, decryption transparencies are not convenient for voters, which already have to carry a smartcard and a reader, a single decryption transparency is not likely to be a ubiquitous solution, since it may not work well with all displays, and keys and transparencies make voters vulnerable to coercion.

(Gobioff et al., 1996) discusses minimal properties necessary for secure input or output of a smartcard in a point-of-sale (POS); a POS is comparable to our hosting computer but using an ordinary smartcard reader without human I/O capabilities. They discuss additional I/O capabilities that smartcards could have and how they could be used to address security problems raised by hostile POS environments. The aspects more relevant for our work are, using their terminology, privacy of the output and general trusted output.

Privacy of the output is addressed with one-time pad encryption, using a key provided by the smartcard owner using a smartcard input keyboard. However, the decryption of the encrypted output is not addressed, nor even who is going to do so; in our case we could consider visual decryption by the voter using a personal decryption transparency. General trusted output is addressed with trusted input and one bit of trusted output. The method relies on feedback: every output bit is feed back into the smartcard, using the trusted input, and when an error is detected the trusted output bit displays the error condition. In our case a feedback mechanism is possible, but not with the bits of the displayed image. As we will see, we used characters.

Completely Automated Public Turing Tests to Tell Computers and Humans Apart (CAPTCHA (von Ahn et al., 2003)) are ways of transforming text into an image such that only humans can understand it. Attacking computer programs are unable to extract the text from the image due to the properties of the transformation - a hard artificial intelligence (AI) transformation (a problem can be solved by a majority of humans but not by a computer using state-of-the-art AI programs). Consequently, attacking computers also have difficulty to make a useful but undetectable mod- ification to the text within the image. Thus, if the text contains some visual authentication element, it may be used to check the integrity of the image. However, CAPTCHAs are some times hard to understand even by humans and are used to transform single words or small sentences, not long texts, such as electoral ballots. Furthermore, attacking computers may use backend pools of people to interpret CAPTCHAs.

(King and dos Santos, 2005) extends CAPTCHA by introducing keyed AI transformations. These extend text $\rightarrow$ image transformations like CAPTCHA to include a secret shared between the image producer and the authenticator. This way, attacking computers cannot replace entire images based on public contents (e.g. electoral ballots). The examples, however, are with simple, short sentences, and not with long texts, such as electoral ballots.

(Hanley et al., 2005) proposed an e-voting system based on keyed AI transformations. Voters get ballots transformed by CAPTCHAs and should be able to understand them and to make a choice by pointing out a particular section of the ballot (an area of an image, a frame of a film, etc.). The selected section is randomly mapped per voter to a particular answer. Unfortunately, the system has many security problems: the recording of presented ballots and choices made by voters may undermine their privacy and ballots are not authenticated.

\section{VOTING USABILITY ISSUES}

Voting processes raise several usability issues for several reasons (Bederson et al., 2003; Greene et al., 2006; Byrne et al., 2007). First, they are to be used by people with different abilities, such as language understanding, different impairments, such as blindness, etc. In short, the population they are targeted to is very heterogeneous in many aspects, but the system should not disfranchise any voter because of their difference. This means that the usability of voting systems must be very carefully evaluated to detect and eliminate their disfranchising potentials.

Second, usually voters are not trained to use specific voting devices. Many voters effectively deal with voting devices the first time they have to use them in real elections. This may create many usability problems, which are amplified by the reluctance of asking for help (because of privacy concerns), the pressure of long waiting queues, etc.

Its now time to comment our proposal regarding usability issues. First, we are not proposing a perfect voting terminal for being used by everybody in all elections. We are proposing an enhanced, visual interface for a portable, voting terminal. The advantage of using the terminal is that voters may potentially vote anywhere, but that is not mandatory, only possible. Therefore, we do not advocate the use of this terminal for all voters, but only for those willing to get some 
advantage out of it (such as mobility). And, of course, we assume they can read the ballot's text.

Second, this paper presents only one possible interface for the portable terminal, using text. Furthermore, the interface uses images and colors for text authentication, which is not suitable for blind people and creates problems to people with visual impairments, such as color blindness. However, other interfaces may be addressed in the future for helping people with difficulties in using this one (using pictures, audio, braille output interfaces, etc.).

Third, a portable, personal voting terminal allows voters to get used to it, to learn very well how it works and to customize its behaviour in order to facilitate the participation in elections. Therefore, in our work it is relevant to discuss several interface possibilities and their pros and cons, and configuration options, instead of proving a single, inflexible and well-studied interface. This thus not mean that we do not need to carry on a detailed usability study with real voters in realistic elections. However, unlike other voting systems, customization is relevant and should be considered in the interface proposal and also in the training of voters, something that was not considered in (Bederson et al., 2003; Byrne et al., 2007).

Finally, in the interface here presented we deal with the visual authentication of ballot contents. As far as we know, no voting system until now did that; voters assume the system provides them the right ballot, and not a false one. Visual authentication is a task that is natural to increase the cognitive workload of voters, therefore making even more difficult to evaluate the usability of the system. In this document we anticipate some cognitive workload problems, some of them detected with practical experience with users, and we draw some possible solutions to deal we them. Again, our goal was to provide flexibility to the configuration of the interface in order to better adapt it to the terminal owner. Nevertheless, we assume that voters willing to use this interface for some reason (such as mobility) are aware and comfortable with the extra workload it may introduce in voting processes.

\section{ENHANCED INTERFACE}

We will now present our contribution, a solution for improving the interface of the FINREAD voting terminal without reducing its security. First we describe how filled ballots are presented to voters using the display of hosting computers but without disclosing voters' choices. Next we discuss alternatives for enforcing the integrity control of the presented ballot and we present our preferred solutions.

\subsection{Non-disclosure of Voters' Choices}

The presentation of filled ballots without disclosing voters' choices forced us to look for some way to represent a filled ballot other than traditional ones (e.g. with crosses inside boxes or completed arrows). We used the fact of having two separate displays - the terminal display and the display of the hosting computer - to present complementary contents conveying useful information to the voter only. Naturally, the security of this approach requires that attackers cannot monitor both displays simultaneously.

The link between the information presented in each display is done with numbers. Each possible answer to a question is given a number (hardcoded in the ballot XML or dynamically given by the terminal). When a question and its possible answers are displayed at the hosting computer, the numbers are displayed as well. Simultaneously, on the terminal display are presented only the numbers corresponding to the answers chosen by the voter for that question.

For clarification, here is an example. Let's assume that a question in the ballot has 3 alternatives: YES, NO or none of them (blank), numbered from 0 to 2 (0 for blank). If the voter chooses option NO, the image presented on the screen and the information presented on the terminal will look like shown in Fig. 3.

Multiple choices may be expressed using the same model, requiring only displaying on the terminal more than one number. Considering the question presented in Fig. 4, where the voter can choose up to four answers (Cat, Fish, Dog and Bird), the number of all the chosen ones is presented in the terminal.

For questions with many possible answers, for instance, a number between 0 and 100, the solution is different (see Fig. 5). In this case the image presented by the hosting computer will only contains two choices, one for a blank vote (NO ANSWER) and another for an expressed vote (ANSWER), and the terminal refers the chosen one; but for an expressed vote it will also display the answer (18 in Fig. 5-b).

The voting interface for the voter works like this. The hosting computer displays an image of the ballot containing the current question and the remaining questions that fit in the allocated image space; current question and answers are highlighted (see Fig. 6). The voter reads the current question and chooses one or many answers using the terminal pin-pad. The answers are shown in the terminal as they are expressed; at the end the voter uses a function key of the pin-pad (we used the green key) to confirm the choices and proceed to the next question.

For questions with a single answer from a large set of possible values (as in Fig. 5), the voter first chooses 0 or 1 with the pin-pad, for choosing between a blank vote and an expressed vote. Then, if she chose 1 , she enters a specific answer using the pin-pad. Finally, she confirms the answer, using the confirmation key of the terminal, or repeats the process.

Before committing to the vote with a function key of the pin-pad (we used the yellow key), the voter can browse through the questions, which are highlighted at the hosting computer display, and check the corresponding answers on the terminal display. Browsing 


\begin{tabular}{|c|c|}
\hline & $\begin{array}{l}\text { Tradicional vote } \\
\begin{array}{|l|}\text { Are you a regular voter? } \\
\square \text { YES } \otimes \text { NO }\end{array}\end{array}$ \\
\hline $\begin{array}{l}\text { Vote with F } \\
\text { Terminal }\end{array}$ & $\begin{array}{l}\text { FINREAD terminal and hosting computer } \\
\text { Vote }=2\end{array}$ \\
\hline \begin{tabular}{|l|} 
Computer \\
screen
\end{tabular} & $\begin{array}{lll}\text { Are you a regular voter? } \\
\begin{array}{llll}\text { ()) } & \text { YES } & \text { 2) NO } \\
\end{array}\end{array}$ \\
\hline
\end{tabular}

Figure 3: Voting with a single answer per question (voter chose NO). With the terminal the voter entered 2 for NO.

Tradicional vote
\begin{tabular}{|l|}
\hline Preferred domestic animals? \\
$\otimes$ Cat $\square$ Fish $\quad$ Dog $\quad \square$ Bird \\
\hline
\end{tabular}

Vote with FINREAD terminal and hosting computer

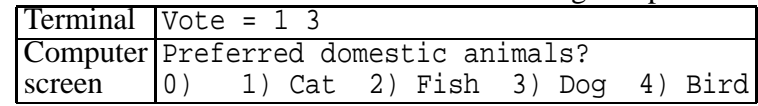

Figure 4: Voting with several answers per question (voter chose Cat and Dog). With the terminal the voter entered 1 and 3 for Cat and Dog, respectively.

Tradicional vote Best year of your life (0-100)?

(a) Vote with FINREAD terminal and hosting computer

\begin{tabular}{|l|l|}
\begin{tabular}{|l|l|}
\hline Terminal & Vote $=0$ (blank) \\
\hline $\begin{array}{l}\text { Computer } \\
\text { screen }\end{array}$ & Best year of your life $(0-100)$ ? \\
0) ANSWER 1) ANSWER
\end{tabular} \\
\hline \multicolumn{2}{|c|}{ Tradicional vote } \\
\hline Best year of your life (0-100)? 18
\end{tabular}

(b) Vote with FINREAD terminal and hosting computer Terminal Vote $=1$ (18)

\begin{tabular}{|l|l|l|}
\hline $\begin{array}{l}\text { Computer } \\
\text { screen }\end{array}$ & Best year of your life $(0-100) ?$ \\
\hline
\end{tabular}

Figure 5: Voting with a single answer from a large set of possible answers per question. In (a) the voter did not vote and in (b) the voter chose 18 . With the terminal the voter entered 0 for a blank vote (a) and 1 and a number for an expressed vote (b).

up and down is performed with a pair of pin-pad function keys (we used keys '*' and '.'). The voter is also able to delete answers, with another function key (we used the red key), while filling the ballot or reviewing her answers. We believe that this reduced set of functionalities is enough for providing a simple and efective browsing through ballot questions and filling and reviewing of chosen answers.

\subsection{Authentication of Displayed Ballots}

Electoral ballots are usually public; therefore attackers may use their information to interfere with the displaying of ballots in tampered hosting computers. Therefore, voters using the terminal must use some mechanism to ensure that the image displayed by the hosting computer was generated by the terminal from a correct XML document.

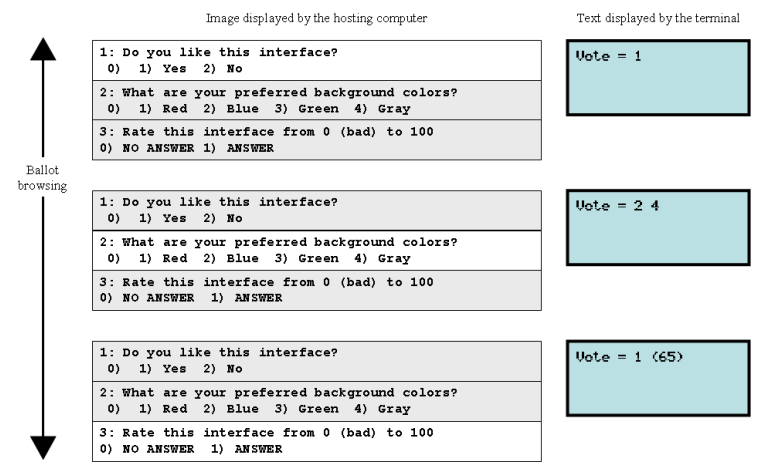

Figure 6: Ballot filling interface. On the left is the image presented by the hosting computer, which changes along the filling process and highlights the current question and all its possible answers. On the right is the text presented by the terminal - the answers to the current question.

The integrity of the XML document is assured by digital signatures; the XML is signed by an electoral Authority and the signature validated by the terminal using the Authority's public key stored inside voters' smartcards (Zúquete et al., 2007). The integrity control of the ballot image is more complex, as it needs to be performed by the voter. This may be accomplished using visual authentication, where the voter, using observation and some helping technology, can ensure that the displayed image if fresh and correct.

To produce visually authenticated images with the terminal we can use voter-provided keys: the voter introduces a key using the terminal pin-pad, and the terminal uses it to authenticate the image. This key may be a long-term key used for visual cryptography or a fresh, random key for a keyed AI transformation.

Another alternative is to use feedback: the terminal produces an image with highlighted, randomly selected characters belonging to the ballot text, which should be feed back in the correct order to the terminal using its pin-pad (active validation). However, for pin-pads such as the one of the FINREAD terminal, which only contains numbers and a few function keys (cf. Fig 2), the introduction of alphabetical characters is not straightforward. One alternative is to map several characters to each key, as in mobile phones; another alternative is to show all the feedback characters, without repetitions and in a different order (e.g. alphabetically) in the terminal display and use the pin-pad to navigate through them and to select the observed highlighted characters in the correct order.

Yet another alternative is to let the terminal to randomly choose keys or feedback characters and to show them using its display. This way, the voter only needs to check if the data displayed by the terminal matches the authentication data existing in the presented image (passive validation).

Hereafter we will describe a solution using feedback characters with active or passive user validation. For each question the terminal chooses a random set 
(a) Passive validation of a feedback string

Image displayed by the hosting computer

1: Do you like this interface?
$\begin{array}{ll}\text { 0) 1) Yes 2) No }\end{array}$

(b) Active validation of a feedback string

Image displayed by the hosting computer

you like this interface?

0) 1) Yes 2) No

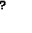

Uote $=1$

Red = thllelonts:

Figure 7: Visual authentication of displayed questions and answers with colours and a feedback string for (a) passive validation or (b) active validation; the terminal is using red for highlighting feedback characters. With passive validation, the voter checks if the feedback string matches the red characters of the image in the correct order. With active validation, the voter chooses, by navigating with the terminal pin-pad on the alphabetically ordered feedback string, the red characters in the correct order.

of feedback characters from the question and its answers. The feedback characters are then written in the image with some differentiating characteristic, such as a different colour or letter case. The voter identifies the feedback characters in the image and validates them using active or passive validation. For differentiating feedback characters in the images we used colours. Colours, or at least a small set of basic colours, are easy to identify and differentiate by most people, except colour-blind people.

A simple way to use colours to identify feedback characters is the following. First the terminal randomly chooses a highlight colour from a list of basic colours (red, green, blue, yellow, white, black, etc.). The terminal will then compose the text in the image using the highlight colour for the feedback characters and random colours, from the rest of the colour list, for the other characters (see example in Fig. 7). A malicious hosting computer cannot conclude, from the text colours, which are the feedback characters; thus, producing a tampered image for a question and its answers has a probability of success that depends on guessing the actual highlighting colour.

For tampering an image without being noticed, an attacker must guess the highlight colour, identify all the highlighted characters of each image and produce a different, meaningful question and answers using the same ordered set of highlighted characters. Figure 8 shows two different tampered images that can replace the image presented in Fig. 7 and 8-a: image (b) has a different order in the answers and is correctly authenticated if the highlight colours are red or black; image (c) has a different question and is correctly authenticated if the highlight colour is blue.

Assuming that it is feasible to create a convincing tampered image for any highlight colour, the success of a tampering attack basically depends on the number of colours used and on the possibility of creating a single tampered image for more than one colour (as in Fig. 8-b). Thus, using more colours reduces both the probability of guessing the actual highlight colour (a) Original image built with highlight colour red 1: Do you like this interface?

0) 1) Yes 2) No

(b) Tampered answers for red and black highlight colours 1: Do you like this interface?
$\begin{array}{ll}\text { 0) 1) No } & \text { 2) Yes }\end{array}$

(c) Tampered question for the blue highlight colour 1: Do you hate this interface? 0) 1) Yes 2) NO

Figure 8: Original image and tampered images that may be considered valid by the voter.

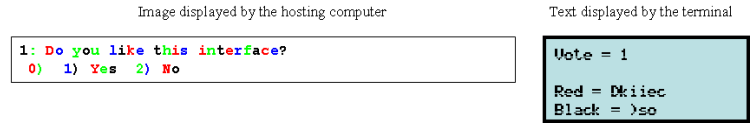

Figure 9: Improved visual authentication of questions and answers with 2 highlight colours (red and black) out of 4 and passive validation of feedback strings.

and the length of the feedback string presented in the voting terminal, improving usability.

On the other hand, the number of colours used and its distribution along the question and answers is also critical to the process of building a convincing tampered image. For instance, using only two colours and alternating them on consecutive characters clearly complicates the task of the attacker for building a convincing image. However, the fewer colours are used, the longer is the feedback string displayed in the voting terminal. Concluding, we have different arguments for using a few or many colours.

A compromise solution is the following. Assuming that there are $N$ possible colours, all of them are used and the terminal chooses 2 highlight colours, one for the question and the other for the answers, possibly the same. For colouring the characters, all $N$ colours are used in blocks of contiguous $N$ characters, but not always with the same order.

With this approach we reduce the probability of correctly guessing the actual highlighting colours from $\frac{1}{N}$ to $\frac{1}{N^{2}}$ without significantly complicating the user interface. Since at least one feedback character appears in each $N$-character block, attackers experience tampering constraints along the entire text. Nevertheless, an attacker may precompute all possible alternative images for any set of highlight colours, which are $N^{L / N}$ images, where $L$ is the number of characters in the original image, and chose the appropriate one with the guessed highlighting colour pair.

Figure 9 shows an implementation of this authentication strategy using only 4 possible colours (RGB plus black) and two highlight colours, thus $N=4$. The two feedback strings are short, with 6 and 5 characters each, being easy to check. The probability of guessing the actual pair of feedback colours is $\frac{1}{16}$ and the number of colouring alternatives is $4^{42 / 4} \approx 10^{6}$, which is high enough for complicating the task of precomputing all possible, convincing tampered images. 


\section{ANALYSIS}

\subsection{Voter Privacy}

If an attacker could monitor the voting terminal of a voter then it could get all the voter's options, destroying this way his privacy. Thus, a fundamental requirement for using correctly the voting terminal is to prevent anyone from monitoring its input interface - the pin-pad. Since the pin-pad is close to the display lines, we can also assume that attackers cannot monitor the terminal display.

Assuming that attackers cannot monitor the terminal interface, then it's straightforward proving that attackers controlling hosting computers cannot get any useful information: displayed ballots have no voters' choices and feedback characters are randomly chosen by terminals. Displayed images gathered by attackers cannot also be used latter to coerce voters to prove how they have voted, because images do not contain any personal information.

\subsection{Image Authentication}

The tampering of displayed images is possible, but the probability of success is small, though not neglectable. This probability depends on two factors: (i) guessing the right set of highlight colours and (ii) producing a convincing image while keeping the ordered sets of highlighted characters. These tasks can be made arbitrarily hard by using high values for the total number of colours $(N)$ and the number of highlight colours $(H)$, but that has also impact on usability. Namely, using high values for $N$ is inconvenient because colours become complex to describe in the terminal (e.g. medium spring green) and hard to match on the displayed image; this last issue is particularly relevant for colour-blind people. Using high values for $H$ is also inconvenient, has it floods the terminal display with feedback strings, ultimately displaying the majority of the characters in the question and answers but in a mangled fashion. Therefore, for usability we should keep $N$ and $H$ as low as possible.

An alternative for reducing the probability of success of a tampering attack is to enable a voter to check on the terminal feedback strings for different colours, but not all at the same time. For instance, if the first presented strings are for red and black, the next strings may use other combinations of colours, like red and green, blue and green, etc. The continuous validation should be triggered by the voter, using a terminal key (we used the key ' $F$ '), and the image displayed should always be the same. The voter may stop at any time for authenticating the displayed image against the feedback strings currently presented on the terminal. This repeated authentication is easy to implement and enables voters to make the authentication mechanism as strong as personally required: the more feedback strings are checked, the higher is the probability of detecting a tampered image.
Colour-blindness raises obvious usability problems for the proposed visual authentication mechanism using colour-based feedback. Allowing voters to continuously change the feedback strings may partially solve the problem for people with limited colour-blindness, as they may change the set of feedback strings until being able to clearly distinguish the two highlighting colours used in the feedback strings. However, for people that only see black and white it simply should not work; for those people another visual authentication mechanism must be used.

\subsection{Feedback Validation}

We referred two possible policies that can be used to visually authenticate images using feedback characters - passive validation or active validation. With passive validation, voters mentally check the highlighted characters against the feedback string and conclude if the image is genuine or a fraud. On the contrary, with active validation, voters have to use the pin-pad to input the highlighted characters and the terminal checks them against the feedback strings.

Passive validation is more convenient for users but also more prone to human errors. Careless voters may proceed with the voting without properly authenticating the images or even proceed after detecting many consecutive display errors. Furthermore, voters must be conscious about error management, i.e., if a voter gets 6 errors in 7 authentication attempts, then there is an obvious problem with the displayed images and the voter should not further consider them in the current voting process. If, on the contrary, the voter gets 1 error in the same 7 attempts, then probably the error was caused by an occasional misinterpretation of feedback elements.

Active is less convenient to voters but is more secure, because voters can not proceed without properly authenticating the image. But some escape mechanism should exist to prevent denial of service attacks by hosting computers or visual problems with voters; for instance, after a limited sequence of authentication errors the terminal should abandon the presentation of images and use only its small display to show contents to the voter. Active feedback is also less convenient for consecutive authentication with different colours. This means that voters may tend to minimize the input feedback to proceed with the voting process, thus reducing the overall authentication strength.

Concluding, both policies have pros and cons: for voter convenience we should go for passive validation, for voter security we should go for active validation. We expect that usability tests may introduce some bias towards each of these policies.

\subsection{Preliminary Usability Experiences}

For evaluating the usability of this voting interface with many people we developed a Java applet demon- 
strator $^{1}$. This demonstrator contains a replica of the FINREAD terminal and the image presented by the hosting computer and implements only passive feedback. Furthermore, it allows voters to choose the set of colours used for painting the actual question and its answers. This dynamic colour palette enables voters to learn the set of colours they feel comfortable with, in order to ultimately customize their smartcard for controlling their FINREAD terminal.

Preliminary experiences showed that scattered colouring of isolated letters introduces a very high cognitive load in the authentication task. Furthermore, long questions or many answers require many colours, because feedback strings have a limited length, which further complicates the location of the feedback characters.

A solution for this problem is aggregation of characters with the same colour. Aggregation facilitates significantly the location of the coloured characters being used in the authentication process, because the text to authenticate becomes coloured by "areas". Furthermore, aggregation improves the readability of text when using many different colours The demonstrator allows voters to change the level of aggregation to evaluate its impact in the cognitive load involved in ballot authentication operations

Changing the colour associated with the feedback string, without changing the image presented by the hosting computer, enables the voter to validate as many "areas" of the presented question and answers as she feels required, in order to increase her confidence in the authentication. High aggregation levels and short questions, or sets of answers, such as yes/no answers, may even produce feedback strings with all the text to authenticate.

\section{CONCLUSIONS}

In this paper we described a proposal for enhancing the interface of an FINREAD-based, portable voting terminal. This interface presents ballot images in the hosting computer display, but prevents the hosting computer to violate the voter privacy or to deceive him with fake images without being noticed.

The design of the enhanced interface relies on the fact that voters have two different displays: one protected, on the terminal, and another insecure one, provided by the hosting computer. The terminal display shows small amounts of information allowing the voter to check his answers and verify the correctness of the presented image. For verifying the correctness of the image a different kind of visual authentication is used, based on random, coloured feedback characters. Even with a small number of colours it is possible to reduce to acceptable levels the probability of deceiving a voter with tampered images. Anyway, this (small) probability can be arbitrary reduced by

\footnotetext{
${ }^{1}$ http://www.ieeta.pt/ravz/FINREAD
}

allowing the voter to require many different, consecutive visual authentications for each image.

The implementation of this enhanced interface is in the beginning. First we will use the demonstrator to conduct usability tests in order to evaluate its problems and limitations. For this purpose, we plan to improve the demonstrator to handle either passive or active feedback and to introduce errors in the image presented in the hosting computer. Only afterwards we will add it to the current REVS TCB, using the FINREAD terminal.

\section{REFERENCES}

Bederson, B. B., Lee, B., Sherman, R. M., Herrnson, P. S., and Niemi, R. G. (2003). Electronic voting system usability issues. In Proc. of the SIGCHI Conf. on $\mathrm{Hu}$ man Factors in Computing Systems (CHI'03), pages 145-152, Ft. Lauderdale, FL, USA. ACM.

Byrne, M. D., Greene, K. K., and Everett, S. P. (2007). Usability of voting systems: baseline data for paper, punch cards, and lever machines. In Proc. of the ACM SIGCHI Conf. on Human Factors in Computing Systems (CHI'07), pages 171-180, San Jose, CA, USA.

FINREAD Consortium(2003). FINREAD Technical Specifications, Parts 1-8.

Gobioff, H., Smith, S., Tygar, J. D., and Yee, B. (1996). Smart Cards in Hostile Environments. In 2nd USENIX Works. on Electronic Commerce, Oakland, USA.

Greene, K. K., Byrne, M. D., and Everett, S. P. (2006). A comparison of usability between voting methods. In Proc. of the 2006 USENIX/Accurate Electronic Voting Technology Works., pages 2-2, Vancouver, B.C., Canada. USENIX Association.

Hanley, D., King, J., and dos Santos, A. (2005). Defeating Malicious Terminals in an Electronic Voting System. In Proc. of 5th Brazilian Symp. on Information and Computer System Security (SBSeg 2005), Florianópolis, $\mathrm{SC}$, Brazil.

Joaquim, R., Zúquete, A., and Ferreira, P. (2003). REVS A Robust Electronic Voting System. IADIS Int. Journal of WWW/Internet, 1(2).

King, J. and dos Santos, A. (2005). A User-Friendly Approach to Human Authentication of Messages. In Financial Cryptogr. and Data Security. LNCS 3570.

M., M. N. and Shamir, A. (1995). Visual Cryptography. In Eurocrypt '94. Springer-Verlag. LNCS 950.

Naor, M. and Pinkas, B. (1997). Visual Authentication and Identification. In Advances in Cryptology - Crypto 97 Proc. Springer-Verlag. LNCS 1294.

von Ahn, L., Blum, M., Hopper, N. J., and Langford, J. (2003). CAPTCHA: Using Hard AI Problems For Security. In Adv. in Cryptology - Eurocrypt 2003 Proc. Springer-Verlag. LNCS 2656.

Zúquete, A., Costa, C., and Romão, M. (2007). An Intrusion-Tolerant e-Voting Client System. In 1st Works. on Recent Advances on Intrusion-Tolerant Systems (WRAITS 2007), Lisboa, Portugal. 